\title{
Acute Pneumonia: What Infection We're Treating?
}

\author{
I Klepikov* \\ Pediatric Surgeon, USA \\ *Corresponding author: Klepikov Igor, Pediatric Surgeon, USA
}

Submission: 㘹 October 12, 2018; Published: 眥 November 13, 2018

\section{Opinion}

Dear colleagues, the assessment of the events and phenomena taking place around us, as well as the nature of the reaction to them are determined by the existing ideas and views on the essence of what is happening. As the old postulate of philosophy says: "Ideas rule the world". This Maxim is relevant for all spheres and branches of our life, including medicine. In the latter case, conceptual ideas about the nature of the disease determine the direction and nature of medical care. It is no secret that the deeper and more specific our knowledge about the causes and mechanisms of the development of the painful process, the higher the chances of achieving maximum success in its treatment. In this context, the modern understanding of the nature of acute pneumonia (AP) raises many questions, to which there are actually no reasoned answers in the available literature.

The long history of the study of AP can be divided into two periods. The conditional line between these rounds is the beginning of the clinical use of antibiotics. The first fantastic results of treatment with op antibiotics formed the opinion that these drugs may well be the only way to help such patients. In the first years of antibiotic therapy, confident results of treatment of AP did not cause any doubts in such approaches. In addition, there is a growing belief that the main problem of $\mathrm{AP}$ is a microbial pathogen and therefore the main purpose of treatment should be its suppression. Thus, the microbiological characterization of AP formed the basis of the concept of the disease and is now often its only description. Description of the Microbiology of AP usually includes a small group of non-specific pathogens, although to date the possibility of participation in this process of more than 100 different microorganisms has been proved [1]. The constant influence of such information on the judgments about the nature of inflammation in the lungs has already led to the fact that AP is increasingly designated and classified as an infectious disease, although it has never been and is not an infectious disease in the full sense of the word.

All this information could not be mentioned if the effectiveness of antibiotic therapy and the results of treatment of patients with AP remained at the level of primary. However, there have been many fundamental changes in recent decades that are already causing legitimate concern and forcing us to seek solutions to growing problems. Today, it is difficult to determine the generation number of antibiotics, which is currently the most popular and effective. At the same time, the duration of antibiotic use in many patients has increased significantly and is a complex alternation of drugs of different groups. Widespread and prolonged use of antibiotics has led to the emergence and gradual increase in the list of antibioticresistant strains. The same reasons are the basis for the periodic change of leaders among the causative agents of acute pneumonia. In such a situation, it is impossible to assess the practical benefits of modern views on the nature of the AP. The vast majority of patients with AP have been cured and continue treatment without checking the microbiological diagnosis of the disease. The exception is only a small percentage of patients, who are being treated in a hospital because of purulent complications of the process and in which cultures of the material for bacteriological examination were selected directly from the zone of inflammation. But this approach to diagnosis comes too late to clarify the choice of antimicrobial drug and expect to achieve an early effect.

From my point of view, modern concepts of acute pneumonia, which are mainly focused on the microbial factor, are rather a brake in assessing the causes and development of AP than a real help in studying and deepening these ideas. Currently, many features of AP development are explained exclusively from the point of view of Microbiology of the process. For example, the progression of inflammation in the lungs and the development of complications, despite the use of the most modern drugs and treatments, is usually interpreted as the presence of a particularly virulent infection. But, try to find objective evidence of this point of view and you will not be able to find them. Where, when and by whom it is proved that in this category of patients the causative agent of AP differs in special aggression? Or another example, when a narrow view of the nature of the disease excludes a reasoned explanation of the causes of the so-called sterile pleural empyema.

The paradox of the current situation is that every doctor (I have no doubt) is guided by the following axioms of medical science. In particular, AP is the only form of acute nonspecific inflammation that develops in the region of the small circle of blood circulation. Small and large circles of blood circulation, having a close anatomical connection with each other, have the opposite interdependence 
in the regulation of blood flow. From this position, the automatic transfer of traditional methods of intensive therapy (for example, intravenous infusion of solutions) to help patients with AP requires caution and objectivity of testing. The list of such paradoxes and oddities in the current state of the problem of AP should not be continued, they have been mentioned in a number of my previous publications, and these copies can be easily found in publications of the last two years. The purpose of this message is to draw the attention of readers once again to the continuing lack of progress in addressing the issue of AP. There is no doubt that among the readers there are specialists who will not only be able to critically assess the existing dogmas and understand the essence of mistakes, but also to continue to revise the approach to solving this problem. Moreover, the first practical steps in this direction were not only made, but also successfully tested on heavy clinical material. For many years, the report on the results of this work could only be found in Russian [2]. However, these materials, published in English [3], are now available and can serve as a starting point for future research for a wide range of professionals.

\section{References}

1. https://en.wikipedia.org/wiki/Community-acquired_pneumonia

2. Klepikov I (1989) Acute pneumonia and its' purulent-destructive complications in children in conditions of large industrial center of Western Siberia. Author's summary of Doctor's Abstracts. Saint-Petersburg, USSR.

3. Klepikov I (2017) Acute pneumonia: a new look at the old problem. Lambert Academic Publishing, ISBN (978-3-330-35250-6).
Creative Commons Attribution 4.0 International License

For possible submissions Click Here

\section{Submit Article}

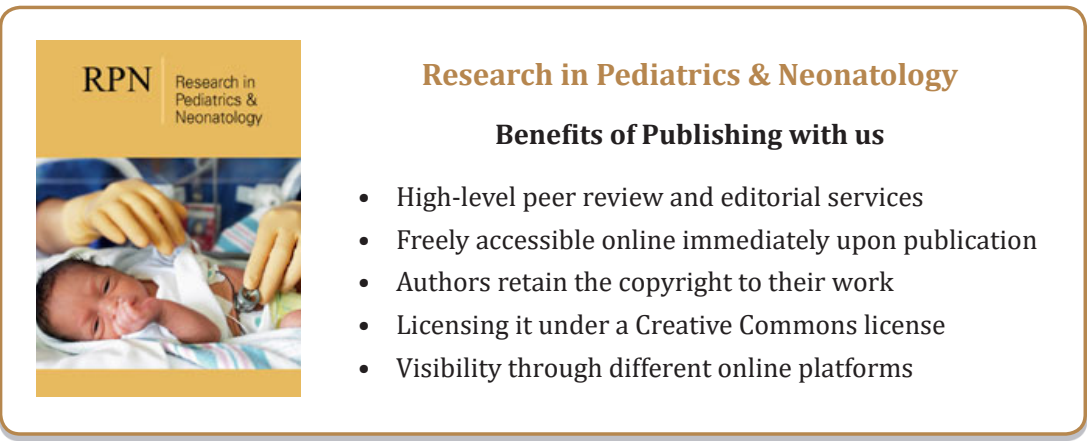

